\title{
PERTURBATION FORMULAE FOR THE SCHRÖDINGER OPERATOR WITH A PERIODIC POTENTIAL NEAR PLANES OF DIFFRACTION
}

\author{
Yu. E. KARPESHINA
}

\begin{abstract}
Perturbation formulae for Bloch eigenvalues and their spectral projections in a vicinity of the von Laue diffraction planes are constructed. They are converging series with respect to a model operator, roughly taking into account the diffraction inside the crystal. The behaviour of the isoenergetic surface near the von Laue diffraction planes is described.
\end{abstract}

\section{Introduction}

The motion of a particle in a bulk matter is often described by the Schrödinger operator with a periodic potential. Therefore, it is interesting to have a detailed mathematical analysis of its spectral properties, in particular, to construct perturbation formulae for its eigenvalues and eigenfunctions.

Thus, we consider the operator

$$
H=-\Delta+V
$$

in $L_{2}\left(R^{3}\right)$, where $V$ is the operator of multiplication by a periodic potential, a trigonometric polynomial.

In $[1],[2]$, perturbation series for Bloch eigenvalues and spectral projections were constructed on a rich set of quasimomenta. On this set, the perturbed and unperturbed eigenvalues and spectral projectors turns out to be close. Our aim here is to study the case when there exists a real refraction inside the crystal, i.e., when the perturbation is significant. Namely, we construct perturbation formulae for quasimomenta in a vicinity of the von Laue diffraction planes. We describe the Bloch eigenvalues and their spectral projections of $H$, which are close to those of a model operator, roughly taking into account the refraction inside the crystal.

To simplify notation we consider the case where the cell of periods is orthogonal. All arguments go through, with obvious modifications, for nonorthogonal lattices. We use a representation of the potential $V(x)$ in

Received May 16, 1994; revised December 1, 1994. 
the form :

$$
V(x)=\sum_{m \in Z^{3},|m|<R_{0}} v_{m} \exp i\left(\vec{p}_{m}(0), x\right)
$$

where $0<R_{0}<\infty,(\cdot, \cdot)$ is the inner product in $R^{3}$ and $\vec{p}_{m}(0)$ is a vector of the dual lattice:

$$
\vec{p}_{m}(0)=2 \pi\left(m_{1} a_{1}^{-1}, m_{2} a_{2}^{-1}, m_{3} a_{3}^{-1}\right) .
$$

The potential $V$ is real, so $v_{m}=\bar{v}_{-m}$. It may be assumed with no loss of generality that $v_{0}=0$.

I. M. Gelfand [3] showed that the investigation of the spectral properties of $H$ reduces to the study of a family of regular operators $H(t), t \in K$, where $K$ is the elementary cell of the dual lattice,

$$
K=\left[0,2 \pi a_{1}^{-1}\right) \times\left[0,2 \pi a_{2}^{-1}\right) \times\left[0,2 \pi a_{3}^{-1}\right) .
$$

The vector $t$ is called the quasimomentum. Let $Q$ be the elementary cell of the periods of the potential- $Q=\left[0, a_{1}\right) \times\left[0, a_{2}\right) \times\left[0, a_{3}\right)$. The operator $H(t), t \in K$, acts in $L_{2}(Q)$. It is described by formula (1) and the quasiperiodic conditions:

$$
\begin{aligned}
& u\left(a_{1}, x_{2}, x_{3}\right)=\exp \left(i t_{1} a_{1}\right) u\left(0, x_{2}, x_{3}\right), \\
& u\left(x_{1}, a_{2}, x_{3}\right)=\exp \left(i t_{2} a_{2}\right) u\left(x_{1}, 0, x_{3}\right), \\
& u\left(x_{1}, x_{2}, a_{3}\right)=\exp \left(i t_{3} a_{3}\right) u\left(x_{1}, x_{2}, 0\right) .
\end{aligned}
$$

The first derivatives with respect to $x_{j}, j=1,2,3$, must also satisfy similar conditions.

The operator $H(t)$ has a discrete spectrum $\Lambda(t)$ :

$$
\Lambda(t)=\cup_{n=1}^{\infty} \lambda_{n}(t), \lambda_{n}(t) \underset{n \rightarrow \infty}{\longrightarrow} \infty .
$$

The spectrum $\Lambda$ of the operator $H$ is the union of the spectra of the operators $H(t)$ :

$$
\Lambda=\cup_{t \in K} \Lambda(t)=\cup_{n \in N, t \in K} \lambda_{n}(t) .
$$

The functions $\lambda_{n}(t)$ are continuous, so $\Lambda$ has a band structure:

$$
\Lambda=\cup_{n=1}^{\infty}\left[q_{n}, Q_{n}\right], q_{n}=\min _{t \in K} \lambda_{n}(t), Q_{n}=\max _{t \in K} \lambda_{n}(t) .
$$

The eigenfunctions of the operator $H$ are obtained by quasiperiodic continuation (see (3)) of the eigenfunctions of all the operators $H(t)$ to $R^{3}$.

Let $H_{0}(t)$ be the operator corresponding to the zero potential. Its eigenfunctions are the plane waves:

$$
\exp \left(i\left(\vec{p}_{j}(t), x\right)\right), j \in Z^{3}, \vec{p}_{j}(t)=\vec{p}_{j}(0)+t .
$$


The eigenfunction (4) corresponds to the eigenvalue $p_{j}^{2}(t) \equiv\left|\vec{p}_{j}(t)\right|^{2}$. Thus, the spectrum of the free operator $H_{0}$ is:

$$
\Lambda_{0}(t)=\left\{p_{j}^{2}(t)\right\}_{j \in Z^{3}} .
$$

Using the basis (4) we write the matrix of $H(t)$ in the form

$$
H(t)_{m j}=p_{m}^{2}(t) \delta_{m j}+v_{m-j},
$$

where $\delta_{m j}$ is the Kronecker symbol.

In the physics literature, the important concept of the isoenergetic surface $S_{0}(k)$ of the free operator is used [4]. This is the set of $t$ in $K$, for which the free operator $H_{0}(t)$ has an eigenvalue equal to $k^{2}$. It is obvious that $t \in S_{0}(k)$ if and only if $p_{m}^{2}(t)=k^{2}$ for some $m \in Z^{3}$. The set $S_{0}(k)$ is obtained by partitioning the sphere of radius $k$ centred at zero into "titles" by the dual lattice. After this, the "titles" are translated in a parallel manner into $K$. Let $S_{1}(k)$ be a subset of $S_{0}(k)$. We say that $S_{1}(k)$ has an asymptotically full measure on $S_{0}(k)$ if

$$
\frac{s\left(S_{1}(k)\right)}{s\left(S_{0}(k)\right)} \underset{k \rightarrow \infty}{\longrightarrow} 1
$$

here and below $s(\cdot)$ is the surface area.

In [1], a nonsingular set $S(k, V, \delta) \subset S_{0}(k)$ was constructed so that for any point $t$ of the $\left(k^{-2-2 \delta}\right)$-neighbourhood of it, the perturbed and unperturbed spectral projections and eigenfunctions are close-more precisely, the corresponding perturbation series converge. The nonsingular set has an asymptotically full measure on $S_{0}(k)$. However, besides the nonsingular set, there exists also the singular part of $S_{0}(k)$, where perturbed and unperturbed eigenvalues and eigenfunctions are, generally speaking, not close. For example, let us consider the plane wave $\exp i\left(\vec{p}_{j}(t), x\right)$, such that its momentum $\vec{p}_{j}(t)$ satisfies the von Laue diffraction conditions, i.e., $p_{j}(t)=p_{j+q}(t)$ for some $q \neq 0$. It is well-known that this wave is refracted by the crystal, the refracted wave being

$$
a \exp i\left(\vec{p}_{j+q}(t), x\right), \quad a \in R .
$$

The latter wave interferes with the initial one and distorts it strongly. Hence, the perturbed and unperturbed eigenfunctions obviously are not close when $t$ satisfies the von Laue diffraction conditions. Thus, the existence of the singular set is connected with diffraction conditions-in the most simple situation with, the von Laue diffraction conditions.

In the two- and three-dimensional situations, the unstable case was studied by J. Feldman, H. Knorrer and E. Trubowitz [5]. In the threedimensional case, they study the eigenvalues of $H$, which are not close 
to the unperturbed ones, but can be approximated by eigenvalues of the operator $-\Delta+V_{\gamma}$, where $\gamma$ is some vector of the dual lattice and $V_{\gamma}$ is independent of $x$ in the direction $\gamma$, i.e.,

$$
V_{\gamma}(x)=V_{\gamma}\left(x-\gamma(x, \gamma)|\gamma|^{-2}\right)=\sum_{j:\left(\vec{p}_{j}(0), \gamma\right)=0} v_{j} \exp i\left(x, \vec{p}_{j}(0)\right) .
$$

It was proved that for arbitrary $\gamma$ of the dual lattice and any eigenvalue of $H_{\gamma}(t)$ corresponding to a sufficiently large momentum in the direction $\gamma$, there exists a close eigenvalue of the operator $H(t)$ with the same quasimomentum, multiplicity being taken into account. The same result was proved for $n=2$. Moreover, in the two-dimensional case, it was shown that, on the rich set of $t$, the corresponding eigenfunctions are close too. O. A. Veliev discussed this problem in [6].

We use another approach here, which has its own peculiarities. It provides formulae not only for unstable eigenvalues, but also for their spectral projections in the three-dimensional situation. Converging perturbation series with respect to some model operator roughly describing the refraction are constructed. Having an asymptotic character in a high energy region, they give an infinite number of asymptotic terms. It is proved that these series can be differentiated any number of times with respect to the quasimomentum and preserve their asymptotic character. From the geometrical point of view, we consider the singular set not only as a part of the whole cell $K$, but, more precisely, we consider its "trace" on the isoenergetic surface $S_{0}(k)$ in $K$ of the free operator for a fixed energy $k^{2}$. Namely, we study the perturbations of the eigenvalues $p_{j}^{2}(t)$ and their spectral projections, which satisfy the conditions $k^{2}=p_{j}^{2}(t) \approx p_{j+q}^{2}(t)$, for some given $q$ and $k$. It is proved that for a fixed $q$ and sufficiently large $k$ there exists a rich set of $t$ - an essential part of the singular set - for which perturbation series with respect to the model operator converge. Although the main geometrical problems arises because of fixed $k$ - one has to prove that perturbation series converge on a rich subset of the surface in $K$ - the consideration of the singular set with a fixed energy turns out to be very useful, because it enable us to describe the perturbed isoenergetic surface near the self-intersections of the isoenergetic surface of the free operatorthey obviously are formed by the points satisfying the von Laue diffraction conditions. We can observe how these intersections are transformed into quasi-intersections under the perturbation. This approach also turns out to be effective in resolving a semicrystal problem (see forthcoming papers). We approximate unstable eigenvalues and eigenfunctions of $H$ by those of a model finite-dimensional operator. This operator roughly takes into account the refraction inside a crystal and is simply connected with some 
periodic Schrödinger operator in one-dimensional space.

To clarify the results for the singular set, we first describe the results for the nonsingular set.

\section{Perturbation formulae for the nonsingular set}

It is proved in [1], [2] that there is a rich set of quasimomenta where perturbed and unperturbed eigenvalues and eigenfunctions are close-hence the name nonsingular set. We constructed the nonsingular set $S(k, V, \delta)$ on the isoenergetic surface $S_{0}(k)$ of the free operator., i.e., $S(k, V, \delta) \subset S_{0}(k)$. It is proved that $S(k, V, \delta)$ has an asymptotically full measure on $S_{0}(k)$. To obtain perturbation formulae for $t \in S(k, V, \delta)$, we considered the unperturbed eigenvalue $p_{j}^{2}(t)=k^{2}$. The index $j$ is uniquely defined by the last relation for $t \in S(k, V, \delta)$. Moreover, it is proved that the nearest point $p_{i}^{2}(t), i \neq j$, is on the distance greater than $k^{-1-2 \delta}$ for such t. Then, we consider a circle of radius $(1 / 2) k^{-1-2 \delta}$ centred at the point $z=p_{j}^{2}(t)$. The main result is that a perturbation series for the resolvent converges with respect to some auxiliary operator $\hat{H}$. Note that the series diverges with respect to $H_{0}$, because not only do the von Laue diffraction conditions exist, but also other ones, depending on the potential. From the series for the resolvent, we easily get series for the perturbed eigenvalues and spectral projection. Replacing $\hat{H}$ with the free operator $H_{0}$ in long segments of the series, we obtain simplified formulae valid with a high accuracy. To describe the results rigorously, we construct the auxiliary operator $\hat{H}(t)$. We also will use it for the case of the singular set. We describe the operator $\hat{H}(t)$ for a trigonometric polynomial (2). Let $\Gamma\left(R_{0}\right)$ be the set of $q, q \in Z^{3},|q|<R_{0}$, such that vectors $\vec{p}_{q}(0)$ form a complete subset of linearly independent vectors in the set $\left\{\vec{p}_{m}(0)\right\}_{|m|<R_{0}}$, i.e., $\Gamma\left(R_{0}\right)$ is such that any vector $\vec{p}_{m}(0),|m|<R_{0}$, can be uniquely represented in the form $\vec{p}_{m}(0)=r \vec{p}_{q}(0)$, where $q \in \Gamma\left(R_{0}\right), r \in Z$. It is easy to see that

$$
V=\sum_{q \in \Gamma\left(R_{0}\right)} V_{q}
$$

where $V_{q}$ depends only on $\left(x, \vec{p}_{q}(0)\right)$,

$$
V_{q}=\sum_{r \in Z,|r q|<R_{0}} v_{r q} \exp \operatorname{ir}\left(\vec{p}_{q}(0), x\right) .
$$

Let $0<\delta \ll 1 / 5$. In $Z^{3}$ we consider the subsets:

$$
\begin{gathered}
\Pi\left(k^{1 / 5-8 \delta}\right)=\cup_{q \in \Gamma\left(R_{0}\right)} \Pi_{q}\left(k^{1 / 5-8 \delta}\right), \\
\Pi_{q}\left(k^{1 / 5-8 \delta}\right)=\left\{j:\left|\left(\vec{p}_{j}(0), \vec{p}_{q}(0)\right)\right|<k^{1 / 5-8 \delta}\right\},
\end{gathered}
$$




$$
\begin{array}{r}
T(k, \delta)=\cup_{q, q^{\prime} \in \Gamma\left(R_{0}\right), q \neq q^{\prime}}\left(\Pi_{q}\left(k^{1 / 5-8 \delta}\right) \cap \Pi_{q^{\prime}}\left(k^{3 / 5}\right)\right)=\left\{j: \exists q, q^{\prime} \in \Gamma\left(R_{0}\right),\right. \\
\left.q \neq q^{\prime}:\left|\left(\vec{p}_{j}(0), \vec{p}_{q}(0)\right)\right|<k^{1 / 5-8 \delta},\left|\left(\vec{p}_{j}(0), \vec{p}_{q^{\prime}}(0)\right)\right|<k^{3 / 5}\right\} .
\end{array}
$$

We define the diagonal projection $P_{q}$ and the auxiliary operator $\hat{H}$ as follows:

$$
\begin{gathered}
\left(P_{q}\right)_{j j}=\left\{\begin{array}{l}
1, \text { if } j \in \Pi_{q}\left(k^{1 / 5-8 \delta}\right) \backslash T(k, \delta), \\
0, \text { otherwise; }
\end{array}\right. \\
\hat{H}(t)=H_{0}(t)+\sum_{q \in \Gamma\left(R_{0}\right)} P_{q} V_{q} P_{q} .
\end{gathered}
$$

Let us introduce the notations:

$$
\begin{gathered}
W=V-\sum_{q \in \Gamma\left(R_{0}\right)} P_{q} V_{q} P_{q}, \\
\left.g_{r}(k, t)=\frac{(-1)^{r}}{2 \pi i r} S p \oint_{C_{0}}\left(\left(H_{0}\right)-z\right)^{-1} V\right)^{r} d z \\
G_{r}(k, t)=\frac{(-1)^{r+1}}{2 \pi i} \oint_{C_{0}}\left(\left(H_{0}(t)-z\right)^{-1} V\right)^{r}\left(H_{0}(t)-z\right)^{-1} d z . \\
\hat{g}_{r}(k, t)=\frac{(-1)^{r}}{2 \pi i r} S p \oint_{C_{0}}\left((\hat{H}(t)-z)^{-1} W\right)^{r} d z \\
\hat{G}_{r}(k, t)=\frac{(-1)^{r+1}}{2 \pi i} \oint_{C_{0}}\left((\hat{H}(t)-z)^{-1} W\right)^{r}(\hat{H}(t)-z)^{-1} d z . \\
T(m)=\frac{\partial^{|m|}}{\partial t_{1}^{m_{1}} \partial t_{2}^{m_{2}} \partial_{3}^{m_{3}}}, \quad m \in Z^{3},|m|=m_{1}+m_{2}+m_{3},
\end{gathered}
$$

here and below $C_{0}$ is the circle of the radius $k^{-1-\delta}$ centred at the point $z=k^{2}$. Let $E_{j}$ be the spectral projection of the free operator $H_{0}(t)$ corresponding to an eigenvalue $p_{j}^{2}(t): E_{n m}=\delta_{j n} \delta_{j m}$. The functions $\hat{g}_{r}(k, t)$ and the operator-valued functions $\hat{G}_{r}(k, t)$ are correctly defined in the $\left(k^{-2-2 \delta}\right)$-neighbourhood of the nonsingular set $S(k, \delta, V)$. In [1], the following theorem of expansion into the modified perturbation series is proved. 
Theorem 1. Supposet belongs to the $\left(k^{-2-2 \delta}\right)$-neighbourhood in $K$ of the nonsingular set $S(k, \delta, V), 0<2 \delta<1 / 100$. Then for sufficiently large $k$, $k>k_{0}(V, \delta)$, the interval $\varepsilon(k, \delta) \equiv\left[k^{2}-k^{-1-\delta}, k^{2}+k^{-1-\delta}\right]$ contains a unique eigenvalue of operator $H$. It is given by the absolutely convergent series:

$$
\lambda(t)=p_{j}^{2}(t)+\sum_{r=2}^{\infty} \hat{g}_{r}(k, t),
$$

where the index $j$ is uniquely determined from the relation $p_{j}^{2 l}(t) \in \varepsilon(k, \delta)$. The spectral projection, corresponding to $\lambda(t)$ is determined by the series

$$
E(t)=E_{j}+\sum_{r=1}^{\infty} \hat{G}_{r}(k, t),
$$

which converges in the class $\mathbf{S}_{1}$.

For the functions $\hat{g}_{r}(k, t)$ and the operator-valued function $\hat{G}_{r}(k, t)$, the following estimates are satisfied:

$$
\begin{gathered}
\left|T(m) \hat{g}_{r}(k, t)\right|<m ! k^{-1-\delta-\gamma_{1} r+2|m|(1+\delta)}, \\
\left\|T(m) \hat{G}_{r}(k, t)\right\|_{1}<m ! k^{-\gamma_{1} r+2|m|(1+\delta)}, \\
\gamma_{1}=1 / 15-20 \delta .
\end{gathered}
$$

Furthermore, in [1], it is shown that $\hat{G}_{r}(k, t)=G_{r}(k, t)$, if $r \leq M(k)$ and $\hat{g}_{r}(k, t)=g_{r}(k, t)$, if $r \leq 2 M(k)$, where $M(k)=k^{1-\delta} R_{0}^{-1}$. The next theorem follows from this.

Theorem 2. Under the conditions of Theorem 1, the eigenvalue $\lambda(t)$ and its spectral projection $E(t)$ can be represented in the form

$$
\begin{gathered}
\lambda(t)=p_{j}^{2 l}(t)+\sum_{r=2}^{2 M(k)} g_{r}(k, t)+\varphi(k, t), \\
E(t)=E_{j}+\sum_{r=1}^{M(k)} G_{r}(k, t)+\psi(k, t), \\
M(k)=k^{1-\delta} R_{0}^{-1} .
\end{gathered}
$$

For the functions $g_{r}$ and the operator-valued functions $G_{r}$, estimates (21) and (22) are fulfilled, while for $\varphi(k, t), \psi(k, t)$, the following ones hold:

$$
|T(m) \varphi(k, t)|<m ! k^{-\gamma_{1} R_{0}^{-1} k^{1-2 \delta}} k^{2(1+\delta)|m|},
$$




$$
\|T(m) \psi(k, t)\|_{1}<m ! k^{-\gamma_{1} R_{0}^{-1} k^{1-2 \delta}} k^{2(1+\delta)|m|}
$$

Corollary 1. For the perturbed eigenvalue and its spectral projection, the following estimates are satisfied:

$$
\begin{gathered}
\left|T(m)\left(\lambda(t)-p_{j}^{2}(t)\right)\right|<c m ! k^{-1-\delta-2 \gamma_{1}+2|m|(1+\delta)}, \\
\left\|T(m)\left(E(t)-E_{j}\right)\right\|_{1}<c m ! k^{-\gamma_{1}+2|m|(1+\delta)} .
\end{gathered}
$$

Note that for $r, m$ small enough estimates (21), (22), (27) and (28) can be improved so that there will be only negative powers of $k$ on the right sides.

\section{Perturbation formulae near the planes of diffraction}

In the case of the polyharmonic operator with a periodic potential we constructed, perturbation series with respect to the free operator on the nonsingular set [7] and with respect to a model operator on the singular set [8]. This model operator roughly took into account the diffraction inside the crystal. In the case of the Schrödinger operator, the modification of the perturbation series $\left(H_{0} \rightarrow \hat{H}\right)$ is needed even for the nonsingular set [1]. The series diverges with respect to $H_{0}$, because not only do the von Laue diffraction conditions exist, but also other ones, depending on the potential. Fortunately, it turns out that such modified series converge even on the "simple" part of the singular set, i.e., it is not necessary to reconstruct additionally these series. The "simple" part is proved to be an essential part of the singular set. The perturbation series for the eigenvalue and its spectral projection converge on the "simple" part of the singular set, when we take the operator $\hat{H}(t)$ as the initial one. Note that the operator $H(t)$ has a block structure. The diagonal part of $\hat{H}$ coincides with the corresponding part of the free operator: $\left(I-\sum_{q \in \Gamma\left(R_{0}\right)} P_{q}\right) \hat{H}(t)=(I-$ $\left.\sum_{q \in \Gamma\left(R_{0}\right)} P_{q}\right) H_{0}(t)$. The blocks of $\hat{H}(t)$ are determined by the orthogonal projections $P_{q}, P_{q} \hat{H}(t)=\hat{H}(t) P_{q}=P_{q} \hat{H}(t) P_{q}$. Each block is a "piece" of the matrix of the Schrödinger operator with potential $V_{q}$, i.e., $P_{q} \hat{H}(t) P_{q}=$ $P_{q}\left(H_{0}(t)+V_{q}\right) P_{q}$. Thus, each block is simply connected with the matrix of a periodic Schrödinger operator in the one-dimensional space, because $V_{q}$ depends only on $\left(x, \vec{p}_{q}(0)\right)$. In the case of the nonsingular set, the perturbed eigenvalue $\lambda(t)$ is asymptotically close $(k \rightarrow \infty)$ to an eigenvalue $p_{j}^{2 l}(t)$ of the diagonal part of $\hat{H}(t)$; i.e, $\lambda(t)$ is close to the eigenvalue $p_{j}^{2 l}(t)$ of the free operator $H_{0}(t)$. Accordingly, the spectral projector of $H(t)$ corresponding to $\lambda(t)$ is close to that of $H_{0}(t)$, corresponding to $p_{j}^{2 l}(t)$. We prove that in the case of the singular set-more precisely of its essential part- eigenvalues and spectral projection of $H(t)$ are close to those of the 
block part of $\hat{H}(t)$. We construct the perturbation series for an eigenvalue and its spectral projection on the essential part of the singular set, taking $\hat{H}(t)$ as the initial operator. We obtain this result by constructing the converging perturbation series for the resolvent and integrating it over a small contour. Thus, the blocks $P_{q} \hat{H} P_{q}=P_{q}\left(H_{0}(t)+V_{q}\right) P_{q}$ describe roughly the refraction inside the crystal for $t$ of the essential part of the singular set.

The proof of the formulae contains the analytical and geometrical parts. In the analytical part, we formulate the conditions of the convergence of the series in the form of inequalities for quasimomentum $t$ (see (34)-(39)). Note that these conditions are similar to those for the nonsingular set up to the replacement of an eigenvalue $p_{j}^{2}(t)$ of the diagonal part of $\hat{H}(t)$ by an eigenvalue of the block part. In the geometrical part we prove that the conditions are satisfied on the essential part of the singular set. The full proofs will be represented in a forthcoming paper. Here we note only that the analytical part is similar to that for the nonsingular set up to the replacement mentioned above. The geometrical part is much more technical. The singular set is only a small part of the isoenergetic surface of the free operator. Therefore, to prove that the conditions of convergence can be satisfied on it, one has to make more subtle considerations than in the case of the nonsingular set.

Thus, we consider the spherical layer on the sphere $|x|=k$ :

$$
S_{q}(k,-\delta)=\left\{x:|x|=k,\left.|| x\right|^{2}-\left|x+\vec{p}_{q}(0)\right|^{2} \mid<k^{\delta}\right\} .
$$

Let $\mathcal{K}$ be the mapping

$$
\mathcal{K}: R^{3} \rightarrow K, \quad \mathcal{K} \vec{p}_{j}(t)=t .
$$

It is easy to see that the isoenergetic surface of the free operator $S_{0}(k)$ is given by the formula $S_{0}(k)=\mathcal{K} S_{k}$, where $S_{k}$ is the sphere $|x|=k$. It is clear that the translation $\mu_{q}(k, \delta) \equiv \mathcal{K} S_{q}(k,-\delta)$ of the set $S_{q}(k,-\delta)$ to the elementary cell of the dual lattice $K$ belongs to the isoenergetic surface $S_{0}(k)$ of the free operator $H_{0}$, but does not belong to the nonsingular set, described in [1], where perturbed and unperturbed eigenvalues are proved to be close. Next, we denote the eigenvalues of $\hat{H}$ (see (12)) as $p_{j}^{2}(t)+\Delta \hat{\Lambda}_{j j}(t), \Delta \hat{\Lambda}_{j j}(t)$ depending continuously on $t$ for every $j$. It is clear that $\Delta \hat{\Lambda}_{j j}=0$, when $j \notin \Pi_{q}\left(k^{1 / 5}\right) \backslash T(k, \delta)$. Let us consider also the part $\hat{\mu}_{q}(k, \delta)$ of the isoenergetic surface of $\hat{H}$ situated in the $\left(c k^{-1}\right)$ neighbourhood of $\mu_{q}(k, \delta)$. It is described by the formula:

$$
\hat{\mu}_{q}(k, \delta)=\left\{t: \exists j, j \in Z^{3}, p_{j}^{2}(t)+\Delta \hat{\Lambda}_{j j}(t)=k^{2},\left|p_{j}^{2}(t)-p_{j+q}^{2}(t)\right|<k^{\delta}\right\} .
$$


In fact, $\hat{\mu}_{q}(k, \delta)$ is very close to the corresponding part of the isoenergetic surface of the operator $H_{q}=-\Delta+V_{q}$, because $p_{j}^{2}(t)+\Delta \hat{\Lambda}_{j j}(t)$ coincides with an eigenvalue $p_{j}^{2}(t)+\Delta \Lambda_{j j}(t)$ of $H_{q}$ up to a value of order $k^{-c k^{1 / 5-8 \delta}}$ (see [1]). Thus, all of the following assertions are also valid for $\hat{\mu}_{q}(k, \delta)$ which is the corresponding part of the isoenergetic surface of $H_{q}$.

Note that $\hat{\mu}_{q}(k, \delta)=\mathcal{K} \hat{S}_{q}(k,-\delta)$, where

$$
\hat{S}_{q}(k,-\delta)=\left\{x:|x|^{2}+\varphi_{0}(x)=k^{2},\left.|| x\right|^{2}-\left|x+\vec{p}_{q}(0)\right|^{2} \mid<k^{\delta}\right\},
$$

$\varphi_{0}(x)$ being uniquely determined from the formulae

$$
\varphi_{0}(x)=\Delta \hat{\Lambda}_{j j}(t), \quad x=\vec{p}_{j}(t) .
$$

It is not hard to show that $\hat{S}_{q}(k,-\delta)$ is the union of curve cylinders, because $\Delta \hat{\Lambda}_{j j}$ depends only on the projection of $\vec{p}_{j}(t)$ on $\vec{p}_{q}(0)$. Note that $\Delta \hat{\Lambda}_{j j} \neq 0$ in (30), because $j \in \Pi_{q}\left(k^{1 / 5}\right)$. We construct the perturbation series with respect to $\hat{H}(t)$. In the case of the nonsingular set, the corresponding pieces of the isoenergetic surfaces of $H_{0}(t)$ and $\hat{H}(t)$ coincide. Clearly, describing the nonsingular set on the isoenergetic surface of one of them, we, of course, describe it for the other too. In the case of the nonsingular set, these surfaces are not the same, i.e., $\mu_{q}(k, \delta)$ does not coincide with $\hat{\mu}_{q}(k, \delta)$. Therefore, the question is : what isoenergetic surface to describe the simple part of the singular set on? Since we construct the perturbation series with respect to $\hat{H}(t)$, it is natural to describe this set on $\hat{\mu}_{q}(k, \delta)$. We formulate the corresponding geometric lemma here. Moreover, we describe the simple part of the nonsingular set on $\mu_{q}(k, \delta)$ too. However, the first description is more convenient for applications. Thus, we shall show that there exists a subset $\hat{\chi}_{q}^{0}(k, V, \delta)$ of an asymptotically full measure on $\hat{\mu}_{q}(k, \delta)$, such that for $t \in \hat{\chi}_{q}^{0}(k, V, \delta)$ the perturbation series with respect to $\hat{H}(t)$ converge. For such a $t$, the operator $\hat{H}(t)$ has an eigenvalue, which can be represented in the form $p_{j}^{2}(t)+\Delta \hat{\Lambda}(t)_{j j}$, j being uniquely determined from the relation $p_{j}^{2}(t)+\Delta \hat{\Lambda}(t)_{j j}=k^{2}$. The series for $H(t)$ converge with respect to $\hat{H}(t)$. Now we give the formulation of the main geometric lemma.

Lemma 1. Let $0<\delta<1 / 300$. Then for sufficiently large $k, k>k_{0}(V, \delta)$, there exists a set $\hat{\chi}_{q}^{0}(k, V, \delta) \subset \hat{\mu}_{q}(k, \delta)$, such that for any $t$ in its $\left(k^{-2-2 \delta}\right)$ neighborhood there is a unique $j \in Z^{3}$ such that $\left|p_{j}^{2}(t)+\Delta \hat{\Lambda}(t)_{j j}-k^{2}\right|<$ $k^{-1-\delta}\left(p_{j}^{2}(t)+\Delta \hat{\Lambda}(t)_{j j}=k^{2}\right.$ for $t$ of $\left.\hat{\chi}_{q}^{0}(k, V, \delta)\right)$ and the inequality

$$
\left|p_{j}^{2}(t)-p_{j+q}^{2}(t)\right|<k^{\delta}
$$

and the following conditions hold: 
$1^{\prime}$.

$$
\begin{gathered}
\min _{m \in \Omega_{1}}\left|p_{j}^{2}(t)+\Delta \hat{\Lambda}(t)_{j j}-p_{j+m}^{2}(t)\right|>k^{1-10 \delta} \\
\Omega_{1}=\left\{m: m \in Z^{3},|m|<k^{\delta} ; m \neq 0 ; m \neq n_{0} q, n_{0} \in Z\right\} .
\end{gathered}
$$

$2^{\prime}$. For all $m:\left|p_{m+j}^{2}(t)-p_{j}^{2}(t)-\Delta \hat{\Lambda}(t)_{j j}\right|<k^{-20 \delta}, m \neq n_{0} q, n_{0} \in Z$, and $|i|<k^{\delta}, i \neq 0$

$$
\left|p_{m+j+i}^{2}(t)-p_{j}^{2}(t)-\Delta \hat{\Lambda}(t)_{j j}\right|>k^{-12 \delta}\left|p_{m+j}^{2}(t)-p_{j}^{2}(t)-\Delta \hat{\Lambda}(t)_{j j}\right|^{-1} .
$$

$3^{\prime}$.

$$
\min _{m \in Z^{3}, m \neq n_{0} q, n_{0} \in Z}\left|p_{m+j}^{2}(t)-p_{j}^{2}(t)-\Delta \hat{\Lambda}(t)_{j j}\right|>2 k^{-1-\delta} .
$$

$4^{\prime}$. For any $q^{\prime} \in \Gamma\left(R_{0}\right)$ and $m \in \Pi_{q^{\prime}}\left(k^{1 / 5}\right)$, the eigenvalue $p_{m}^{2}(t)+$ $\Delta \hat{\Lambda}(t)_{m m}$ of the operator $\hat{H}_{q}(t)$ lies sufficiently far from the point $p_{j}^{2}(t)+$ $\Delta \hat{\Lambda}(t)_{j j}$, namely,

$$
\left|p_{m}^{2}(t)+\Delta \hat{\Lambda}(t)_{m m}-p_{j}^{2}(t)-\Delta \hat{\Lambda}(t)_{j j}\right|>\left|\left(\vec{p}_{m}(t), \vec{p}_{q^{\prime}}(0)\right)\right|^{-1+\delta} .
$$

$5^{\prime}$. If $m \in T(k, \delta)$, then

$$
\left|p_{m}^{2}(t)-p_{j}^{2}(t)-\Delta \hat{\Lambda}(t)_{j j}\right|>k^{1 / 5-9 \delta} .
$$

The set $\hat{\chi}_{q}^{0}(k, V, \delta)$ has an asymptotically full measure on $\hat{\mu}_{q}(k, \delta)$; moreover the inequality

$$
\frac{s\left(\hat{\mu}_{q}(k, \delta) \backslash \hat{\chi}_{q}^{0}(k, V, \delta)\right)}{s\left(\hat{\mu}_{q}(k, \delta)\right)}<k^{-3 \delta}
$$

holds.

Conditions $1^{\prime}-5^{\prime}$ coincide with conditions $1^{0}-5^{0}$ of the convergence of the series in [1] up to replacement of $p_{j}^{2}(t)$ with $p_{j}^{2}(t)+\Delta \hat{\Lambda}(t)_{j j}$. Note that inequalities (37) and (38) yield that the eigenvalue $p_{j}^{2}(t)+\Delta \hat{\Lambda}(t)_{j j}$ of operator $\hat{H}(t)$ is simple. We denote by $\hat{E}_{j}$ its spectral projection. Let

$$
\hat{g}_{r}^{\prime}(k, t)=\frac{(-1)^{r}}{2 \pi i r} S p \oint_{C_{1}} \hat{A}(t)^{r} d z
$$




$$
\hat{G}_{r}^{\prime}(k, t)=\frac{(-1)^{r+1}}{2 \pi i} \oint_{C_{1}}(\hat{H}(t)-z)^{-1 / 2} \hat{A}^{r}(t)(\hat{H}(t)-z)^{-1 / 2} d z,
$$

where $C_{1}$ is the circle of radius $k^{-1-\delta}$ centred at the point $\lambda=p_{j}^{2}(t)+$ $\Delta \hat{\Lambda}(t)_{j j}$,

$$
\hat{A}(z, t, W)=(\hat{H}(t)-z)^{-1 / 2} W(\hat{H}(t)-z)^{-1 / 2},
$$

$W$ being given by formula (13).

Theorem 3. Suppose $0<\delta<1 / 300, t$ is in the $\left(k^{-2-2 \delta}\right)$-neighbourhood of the set $\hat{\chi}_{q}^{0}(k, V, \delta)$. Then for sufficiently large $k, k>k_{0}(V, \delta)$, there exists a unique eigenvalue of the operator $H(t)$ in the $\left(k^{-1-2 \delta}\right)$-neighbourhood of the point $\lambda=p_{j}^{2}(t)+\Delta \hat{\Lambda}(t)_{j j}$. It is given by the absolutely converging series:

$$
\hat{\lambda}(t)=p_{j}^{2}(t)+\Delta \hat{\Lambda}(t)_{j j}+\sum_{r=2}^{\infty} \hat{g}_{r}^{\prime}(k, t) .
$$

The spectral projection corresponding to $\hat{\lambda}(t)$ is determined by the series:

$$
\hat{E}(t)=\hat{E}_{j}+\sum_{r=1}^{\infty} \hat{G}_{r}^{\prime}(k, t),
$$

which converges in the class $\mathbf{S}_{1}$. For the functions $\hat{g}_{r}^{\prime}(k, t)$ and the operatorvalued functions $\hat{G}_{r}^{\prime}(k, t)$, the following estimates hold:

$$
\begin{gathered}
\left|\hat{g}_{2}^{\prime}(k, t)\right|<k^{-8 / 5+7 \delta}, \\
\left\|\hat{G}_{1}^{\prime}(k, t)\right\|_{1}<k^{-1+4 \delta}, \\
\left\|\hat{G}_{2}^{\prime}(k, t)\right\|_{1}<k^{-7 / 5+6 \delta}, \\
\left|\hat{g}_{r}(k, t)\right|<k^{-1-\delta-\gamma_{1} r}, \\
\left\|\hat{G}_{r}(k, t)\right\|_{1}<k^{-\gamma_{1} r}, \\
\gamma_{1}=1 / 15-20 \delta .
\end{gathered}
$$

\section{Corollary 2.}

$$
\begin{gathered}
\left|\hat{\lambda}(t)-p_{j}^{2}(t)-\Delta \hat{\Lambda}(t)_{j j}\right|<c k^{-1-2 \gamma_{1}}, \\
\left\|\hat{E}(t)-E_{j}\right\|<c k^{-3 \gamma_{1}}, c \neq c(k) .
\end{gathered}
$$


Theorem 4. Under the conditions of Theorem 3, the functions $\hat{g}_{r}^{\prime}(k, t)$ and the operator-valued functions $\hat{G}_{r}^{\prime}(k, t)$ depend analytically on $t$ in the complex $\left(k^{-2-2 \delta}\right)$-neighbourhood of each simply connected component of $\hat{\chi}_{q}^{0}(k, V, \delta)$. They satisfy the estimates:

$$
\begin{gathered}
\left|T(m) \hat{g}_{2}^{\prime}(k, t)\right|<m ! k^{-8 / 5+7 \delta+2(1+\delta)|m|}, \\
\left\|T(m) \hat{G}_{1}^{\prime}(k, t)\right\|_{1}<m ! k^{-1+4 \delta+2(1+\delta)|m|} \\
\left\|T(m) \hat{G}_{2}^{\prime}(k, t)\right\|_{1}<m ! k^{-7 / 5+6 \delta+2(1+\delta)|m|}, \\
\left|T(m) \hat{g}_{r}^{\prime}(k, t)\right|<m ! k^{-1-\delta-\gamma_{1} r+2(1+\delta)|m|}, \\
\quad\left\|T(m) \hat{G}_{r}^{\prime}(k, t)\right\|_{1}<m ! k^{-\gamma_{1} r+2(1+\delta)|m|}
\end{gathered}
$$

Corollary 3. The function $\hat{\lambda}(t)$ and the operator-valued function $\hat{E}(t)$ analytically depend on $t$ in the complex $\left(k^{-2-2 \delta}\right)$-neighbourhood of each simply connected component of the set $\hat{\chi}_{q}^{0}(k, V, \delta)$. They satisfy the following estimates:

$$
\begin{gathered}
\left|T(m)\left(\hat{\lambda}(t)-p_{j}^{2}(t)-\Delta \hat{\Lambda}(t)_{j j}\right)\right| \leq c m ! k^{-1-2 \gamma_{1}+2(1+\delta)|m|}, \\
\left\|T(m)\left(\hat{E}(t)-\hat{E}_{j}\right)\right\|_{1} \leq c m ! k^{-3 \gamma_{1}+2(1+\delta)|m|} .
\end{gathered}
$$

Note that for $r, m$ small enough, estimates (53)-(59) can be improved so that there will be only negative powers of $k$ on the right sides. Theorems 3 and 4 enable us to conclude that the points satisfying the equation $\hat{\lambda}(t)=k^{2}$ are situated near the isoenergetic surface of the operator $\hat{H}$.

Similar results hold for $\mu_{q}(k, \delta)$, which belongs to the isoenergetic surface of the free operator.

Lemma 2. Let $0<\delta<1 / 300$. Then for sufficiently large $k, k>k_{0}(V, \delta)$, there exists a set $\hat{\chi}_{q}^{\prime}(k, V, \delta) \subset \mu_{q}(k, \delta)$, such that for any $t$ in its $\left(k^{-2-2 \delta}\right)$ neighborhood there exists a unique $j \in Z^{3}$ such that

$$
\left|p_{j}^{2}(t)-k^{2}\right|<k^{-1-\delta} \quad\left(p_{j}^{2}(t)=k^{2} \text { for } t \text { of } \hat{\chi}_{q}^{\prime}(k, V, \delta)\right),
$$

inequality (33) and conditions $1^{\prime}-5^{\prime}$ are satisfied. The set $\hat{\chi}_{q}^{\prime}(k, V, \delta)$ has an asymptotically full measure on $\mu_{q}(k, \delta)$. Moreover, the inequality

$$
\frac{s\left(\mu_{q}(k, \delta) \backslash \hat{\chi}_{q}^{\prime}(k, V, \delta)\right)}{s\left(\mu_{q}(k, \delta)\right)}<k^{-3 \delta}
$$

holds. 
The proof of this lemma is similar to that of Lemma 1. Naturally, for $\hat{\chi}_{q}^{\prime}(k, V, \delta)$, the theorems similar to Theorems 3,4 hold:

Theorem 5. Suppose $0<\delta<\frac{1}{300}$ and $t$ is in the $\left(k^{-2-2 \delta}\right)$-neighbourhood of the set $\hat{\chi}_{q}^{\prime}(k, V, \delta)$. Then, for sufficiently large $k$, there exists a unique eigenvalue $\hat{\lambda}(t)$ of operator $H(t)$ in the $\left(k^{-1-2 \delta}\right)$-neighbourhood of the point $\left.p_{j}^{2}(t)+\Delta \hat{\Lambda}(t)_{j j}\right)$. It is given by the absolutely converging series (43), $j$ being uniquely determined by the relation $p_{j}^{2}(t)=k^{2}$. The corresponding spectral projection is given by formula (44).

Corollary 4. If $t$ is in the $\left(k^{-2-2 \delta}\right)$-neighbourhood of the set $\hat{\chi}_{q}^{\prime}(k, V, \delta)$, then estimates (51)-(52) hold.

Theorem 6. Under the conditions of Theorem 5 the functions $\hat{g}_{r}^{\prime}(k, t)$ and the operator-valued functions $\hat{G}_{r}^{\prime}(k, t)$ depend analytically on $t$ in the complex $\left(k^{-2-2 \delta}\right)$-neighbourhood of each simply connected component of the set $\hat{\chi}_{q}^{\prime}(k, V, \delta)$. They satisfy estimates (58).

Corollary 5. The function $\hat{\lambda}(t)$ and the operator-valued function $\hat{E}(t)$ depend analytically on $t$ in the complex $\left(k^{-2-2 \delta}\right)$-neighbourhood of each simply connected component of the set $\hat{\chi}_{q}^{\prime}(k, V, \delta)$. They satisfy the estimates (58), (59).

\section{The behaviour of the isoenergetic surface in a vicinity of the singular set}

Since the eigenvalues and the spectral projections of operator $H$ are close to those of $\hat{H}$ on the essential part of the singular set, the isoenergetic surface of $H$ is close to that of $\hat{H}$. We denote by $\hat{\mu}_{q}(k, t)_{j}$, the part of $\hat{\mu}_{q}(k, \delta)$ corresponding to some given $j$, i.e., a point $t$ belongs to $\hat{\mu}_{q}(k, \delta)_{j}$ if and only if

$$
p_{j}^{2}(t)+\hat{\Delta} \Lambda(t)_{j j}=k^{2} .
$$

Let $\vec{p}_{j \perp}(t)$ be the component of vector $\vec{p}_{j}(t)$ orthogonal to $\vec{p}_{q}(0)$. Equation (61) is resolvable with respect to $\left|\vec{p}_{j \perp}(t)\right|$, since $\Delta \hat{\Lambda}(t)_{j j}$ depends only on $\left(\vec{p}_{j}(t), \vec{p}_{q}(0)\right)$ :

$$
\begin{gathered}
\left|\vec{p}_{j \perp}\right|=f_{0}\left(\left(\vec{p}_{j}(t), \vec{p}_{q}(0)\right)\right) \\
f_{0}\left(\left(\vec{p}_{j}(t), \vec{p}_{q}(0)\right)\right)=\left(k^{2}-\Delta \hat{\Lambda}(t)_{j j}-\left(\vec{p}_{j}(t), \vec{p}_{q}(0)\right)^{2} p_{q}(0)^{-2}\right)^{1 / 2}
\end{gathered}
$$

Thus, any point of the surface $\hat{S}_{q}(k,-\delta)$ can be represented by the formula for a curve cylinder:

$$
x_{\perp}=f_{0}\left(x_{\|}\right), \quad x_{\|}=\left(x, \vec{p}_{q}(0)\right) p_{q}^{-1}(0) .
$$


To obtain $\hat{\mu}_{q}(k, \delta)$, one has to break $\hat{S}_{q}(k,-\delta)$ into pieces by the dual lattice and to translate all pieces into its elementary cell. Let $\hat{e}(t)$ be the normal to $\hat{\mu}_{q}(k, \delta)$. According to Theorem 3 , there exists a subset $\hat{\chi}_{q}^{0}(k, V, \delta)$ of the asymptotically full measure on $\hat{\mu}_{q}(k, \delta)$ on which the perturbation series converges. We denote by $\hat{\chi}_{q}^{0}(k, V, \delta)_{j}$ the intersection of $\hat{\chi}_{q}^{0}(k, V, \delta)$ with $\hat{\mu}_{q}(k, \delta)_{j}$. It turns out that, in the $\left(k^{-2-2 \delta}\right)$-neighbourhood of a piece $\hat{\chi}_{q}^{0}(k, V, \delta)_{j}$ of the isoenergetic surface, there are no other $\hat{\chi}_{q}^{0}(k, V, \delta)_{i}, i \neq j$.

Theorem 7. For $0<\delta<1 / 300$ and sufficiently large $k, k>k_{0}(V, \delta)$, there exists a unique piece $\hat{S}_{H}^{\prime}(k)$ of the isoenergetic surface of $H$ in the $\left(k^{-2-2 \delta}\right)$-neighbourhood of each simply connected component of $\hat{\chi}_{q}^{0}(k, V, \delta)$. In fact, it is in the smaller $\left(k^{-2-\gamma_{1}}\right)$-neighbourhood of $\hat{\chi}_{q}^{0}(k, V, \delta)$ and can be described by the equation

$$
\hat{\lambda}(t)=k^{2}
$$

where the function $\hat{\lambda}(t)$ is determined by the converging series (43). The corresponding spectral projection is given by formula (44). Terms of these series are satisfied by estimates (45)-(49), (56) and (57). The normal $e(t)$, to $S_{H}^{\prime}(k)$ in point $t$ satisfies the asymptotic:

$$
e(t)=\hat{e}(t)+O\left(k^{-2 / 5}\right)
$$

\section{Acknowledgement}

The author expresses deep gratitude to B.S. Pavlov for repeated useful discussions of this work.

\section{References}

1. Yu. E. Karpeshina, Perturbation theory for the Schrödinger operator with a periodic potential, Trudy Mat. Inst. Steklov. 188 (1990); English transl., Proc. Steklov Inst. Math. 3 (1991), 109-145.

2. __ Perturbation Theory formulas for the Scrödinger equation with a nonsmooth periodic potential, Matem. Sbornik 181, 9; English transl. Math. USSR Sbornik 71(1992), 101-122.

3. I. M. Gel'fand, Expansion in eigenfunctions of an equation with periodic coefficients, Dokl. Akad. Nauk SSSR, 73 (1950), 1117-1120 (in Russian).

4. O. Madelung, Festkörpertheorie, Vols. I, II, Springer-Verlag, 1972: English transl., 1978.

5. J. Feldman, H. Knörrer and E. Trubowitz, Perturbatively unstable eigenvalues of a periodic Schrödinger operator, Comment. Math. Helvetici 66 (1991), 557-579.

6. O. A. Veliev, Review by the author of the doctoral thesis, Tbilisi-1989 (in Russian).

7. Yu. E. Karpeshina, Analytic perturbation theory for a periodic potential, Izv. Akad. Nauk SSSR Ser. Mat. 53 (1989), 45-65; English transl. in Math. USSR Izv. 34 (1990). 
8. _ Perturbation series for polyharmonic operator with a periodic potential near planes of diffraction, preprint, ETH, Zürich, 1992.

Institute of Mathematics and Mechanics, Saint Petersburg University Bibliotechnaya 2, Petrodvoretz, 198904, Saint Petersburg, Russia

Current address: Department of Mathematics, University of Alabama at Birmingham, Birmingham, AL 35294-1150

E-mail address: karp@hq.math.lgu.spb.su 